\title{
Metformin and oxyphotodynamic therapy as a novel treatment approach for triple-negative breast cancer
}

\author{
Xiaofeng Pei ${ }^{1,2,3,4}$, Xiaojin Wang ${ }^{1,2,5 \#}$, Jianzhong Xian ${ }^{1,2 \#}$, Jiaoping Mi ${ }^{6}$, Jiebing Gao ${ }^{1,2}$, Xinglin Li $^{1,2}$, \\ Zhijun $\mathrm{Li}^{1,2}$, Min Yang ${ }^{1,2}$, Lei $\mathrm{Bi}^{1,2}$, Yan Yan ${ }^{1,2}$, Weize $\mathrm{Lv}^{3,4}$, Hongjun Jin ${ }^{1,2}$ \\ ${ }^{1}$ Guangdong Provincial Key Laboratory of Biomedical Imaging and Guangdong Provincial Engineering Research Center of Molecular Imaging, \\ The Fifth Affiliated Hospital of Sun Yat-sen University, Zhuhai, China; ${ }^{2}$ Molecular Imaging Center, The Fifth Affiliated Hospital of Sun Yat-sen \\ University, Zhuhai, China; ${ }^{3}$ The Cancer Center, The Fifth Affiliated Hospital of Sun Yat-sen University, Zhuhai, China; ${ }^{4}$ Department of Thoracic \\ Oncology, The Fifth Affiliated Hospital of Sun Yat-sen University, Zhuhai, China; ${ }^{5}$ Department of Cardiothoracic Surgery, The Fifth Affiliated \\ Hospital of Sun Yat-sen University, Zhuhai, China; ${ }^{6}$ Department of Otolaryngology Head and Neck Surgery, The Fifth Affiliated Hospital of Sun \\ Yat-sen University, Zhuhai, China \\ Contributions: (I) Conception and design: H Jin, W Lv; (II) Administrative support: H Jin; (III) Provision of study materials or patients: H Jin, X Pei; \\ (IV) Collection and assembly of data: X Pei, Z Li, X Li, J Xian, J Mi, M Yang, W Lv; (V) Data analysis and interpretation: X Pei, X Wang, J Gao, J \\ Xian,Y Yan, L Bi; (VI) Manuscript writing: All authors; (VII) Final approval of manuscript: All authors. \\ \#These authors contributed equally to this work. \\ Correspondence to: Dr. Hongjun Jin. Guangdong Provincial Key Laboratory of Biomedical Imaging and Guangdong Provincial Engineering Research Center \\ of Molecular Imaging, Molecular Imaging Center, The Fifth Affiliated Hospital of Sun Yat-sen University, Zhuhai, China. Email: jinhj3@mail.sysu.edu.cn; \\ Dr. Weize Lv. The Cancer Center, The Fifth Affiliated Hospital of Sun Yat-sen University, Zhuhai, China. Email: Lvweize@163.com.
}

Background: Treatment for triple-negative breast cancer (TNBC) remains a significant challenge due to a lack of targeted therapies. While photodynamic therapy (PDT) has been utilized as a treatment approach for several types of cancer, oxyphotodynamic therapy (OPDT) is a novel method that improves treatment efficacy by increasing local oxygen concentration. Metformin (MET) has been demonstrated utility as an anti-tumor agent by acting through the adenosine monophosphate-activated protein kinase (AMPK) pathway. We hypothesized that MET in combination with heme, a byproduct of 5 -aminolevulinic acid (ALA), may increase cytotoxicity for cancer treatment. This study aimed to investigate the synergistic effect of MET and ALA with PDT or OPDT on TNBC tumorigenic cells.

Methods: The treatment efficacy and phototoxicity of PDT or OPDT were determined using a cell viability assay. PDT/OPDT experiments were carried out in nine groups based on different combinations and concentrations of ALA and/or MET. To calculate the synergistic effect by compuSyn soft for different groups, cells were incubated with ALA and/or MET at the following concentrations (0, 0.25, 0.5,1, 2, 4, 8, 16, 24, and $32 \mathrm{mM})$. The fluorescence of ALA-induced protoporphyrin IX (PpIX) and MitoTracker Green were observed under a confocal microscope.

Results: The optimized therapeutic concentration ratio of ALA and MET was determined to be 1:1. The inhibition of cancer growth $\left(\mathrm{IC}_{50}\right)$ for each group was 14.03, 10.62, 7.71, 18.27, 22.09, 23.96, 4.57, 10.20, and $8.18 \mathrm{mM}$, respectively. The combination index $(\mathrm{CI})$ values $(\mathrm{fa}=0.5$ ) of the last three combination groups (groups 7, 8, and 9) were 0.44, 1.70, and 1.47, respectively. PpIX fluorescence intensity of group 9 (ALAMET-OPDT group) remained the highest among all groups, indicating an enhanced therapeutic effect.

Conclusions: This study introduces OPDT as a novel anti-tumor therapy for TNBC. Furthermore, the combined use of ALA and MET had a synergistic anti-tumor effect in TNBC cells when combined with OPDT.

Keywords: Oxyphotodynamic therapy (OPDT); metformin (MET); triple-negative breast cancer (TNBC); 5-aminolevulinic acid (ALA)

Submitted Jun 10, 2020. Accepted for publication Sep 08, 2020.

doi: 10.21037/atm-20-5704

View this article at: http://dx.doi.org/10.21037/atm-20-5704 


\section{Introduction}

For decades, light has been used in the treatment of several diseases. The isolation of porphyrins and their localization in tumors, along with the discovery of their phototoxicity on tumor tissue, led to the development of modern photodetection (PD) and photodynamic therapy (PDT) (1). PDT is a therapeutic technique that combines a photosensitizing agent with a light source to generate reactive oxygen species (ROS), which selectively destroy the pathological tissue (2). When the photosensitizers are illuminated, oxygen levels in tissue decrease rapidly and significantly (3). Reduction of tissue oxygen limits the generation of ROS, thus reducing the therapeutic efficacy of PDT (4). Various light sources and nanoparticles can induce tissue reoxidation, but these procedures can be inefficient (5). A large number of studies have focused on third generation photosensitizers, such as hemoglobin and ferric oxide (6-10), which can improve the efficacy of PDT by alleviating hypoxia of the tumor cell microenvironment. Oxyphotodynamic therapy (OPDT) is a high-end novel medical treatment developed in recent years that utilizes light emitting diodes as the light source. Lasers are regarded as an ideal light source due to their single wavelength and high energy level. Using a synchronous light emitting diode and an external oxygen production source, OPDT can overcome the problem of tissue hypoxia, which has traditionally faced PDT. OPDT, like traditional PDT, produces ROS when photosensitizers are exposed to a light source, particularly singlet oxygen free radicals. ROS produced from OPDT affects cellular components, including proteins and DNA, leading to necrosis or apoptosis (11).

The second generation photosensitizer 5-aminolevulinic acid (ALA) is a precursor of endogenous protoporphyrin IX (PpIX), a photosensitizer. PpIX is produced in the mitochondria and is catalyzed by heme synthase in the presence of ALA (12). When the irradiation of the light source matches the absorption spectrum of the photosensitizer, the two ALA molecules are catalyzed by ALA dehydratase to form porphobilinogen and tetrapyridine porphyrin III, a common precursor of all tetrapyrrole substances. This in turn forms protoporphyrin in the mitochondria and binds to iron ions to form ferrous hemoglobin (13).

The main anticancer target of heme is a transcription factor called bach1 (14). Bach1 targets mitochondrial metabolism by inhibiting the transcription of mitochondrial electron transport chain genes (the key source of cellular energy). Metformin (MET) is one of the most widely used oral hypoglycemic drugs globally. In addition to lowering blood sugar, MET also acts on the mitochondria (15). MET inhibits the expression of DNA damage repair factors, which accelerates the apoptosis of cancer cells (16). It can also activate adenosine monophosphate-activated protein kinase (AMPK) to promote the decomposition of lipids, inhibit the synthesis of related proteins, and limit the energy supply of cancer cells, which consequently inhibits cancer cell proliferation and growth (17). Activated AMPK also regulates the expression of downstream factors, leading to cancer cell cycle arrest, apoptosis, and autophagy, ultimately accelerating cancer cell death (18). Furthermore, activated AMPK also inhibits angiogenesisrelated factors and angiogenesis in tumor tissues, thereby blocking the nutritional supply of cancer cells (19). Recent studies revealed that heme sensitizes triple-negative breast cancer (TNBC) tumors to MET by inhibiting bach 1 expression (14). Therefore, the combination of heme and MET may be an important therapeutic treatment approach for TNBC.

TNBC is more prone to recurrence and metastasis compared to other breast cancer subtypes and has fewer treatment options; chemotherapy, therefore, is the main treatment approach (20). PDT is an important and effective method for treating breast cancer recurrence and metastasis (21). However, due to a low amount of PpIX entering mitochondria, TNBC MDA-MB-231 cells are not as sensitive to PDT. Hence, in this study, external oxygen production was added to alleviate hypoxia in cells and increase the efficacy of PDT. This was also combined with MET and ALA to achieve greater therapeutic efficacy for TNBC. We present the following article in accordance with the MDAR reporting checklist (available at http://dx.doi. org/10.21037/atm-20-5704).

\section{Methods}

\section{Reagents and instruments}

ALA was purchased from Sigma-Aldrich (St. Louis, MO, USA). MET was purchased from Beyotime Biotechnology (Haimen, Jiangsu, China). The MTT kit was purchased from Solarbio Science \& Technology (Haidian district, Beijing, China). The OPDT instrument was obtained from Pure Oxygen and Photodynamic Therapy Composite Treating OxyPDT (II) System (Race MBE Co. Ltd., 
Guangzhou, Guangdong province, China). MitoTracker Green FM was obtained from GeneBank (Guangzhou, Guangdong, China).

\section{Cell culture}

The MDA-MB-231 cell line was obtained from the Transformational Medical Center of the Second Affiliated Hospital of Sun Yat-sen University. Cells were cultured in Dulbecco's Modified Eagle's Medium (DMEM) supplemented with $10 \%$ fetal bovine serum (Hyclone, South Logan, UT, USA), $100 \mathrm{U} / \mathrm{mL}$ penicillin (Squirrel Biotechnology, Guangzhou, Guangdong, China) and $100 \mu \mathrm{g} / \mathrm{mL}$ streptomycin (Sigma-Aldrich, St. Louis, MO, USA) and kept at $37{ }^{\circ} \mathrm{C}\left(5 \% \mathrm{CO}_{2}\right)$.

\section{OPDT}

For OPDT, cells were seeded in 96-well plates $(10,000$ cells/well) and grown overnight in complete medium. After incubation with various concentrations of ALA and/or MET $(0,0.5,1,2,4,8,16,24$, and $32 \mathrm{mM})$ at $37^{\circ} \mathrm{C}$ for 4 hours, the cells were irradiated with a $630 \mathrm{~nm}$ laser light. The light source used was a diode laser with a $633 \mathrm{~nm}$ wavelength emission of red light. After irradiation, the cells were incubated with fresh complete medium for 72 hours until further analysis.

\section{Cell viability assay}

An MTT (3(4,5-dimethyl-thiazoyl-2-yl)2,5 diphenyltetrazolium bromide) assay was used to measure the activity of mitochondrial dehydrogenases, which reduces the water soluble tetrazolium salt to a purple insoluble formazan product. The culture medium was removed and replaced with serum-free DMEM $(100 \mu \mathrm{L} /$ well $)$ containing $0.4 \mathrm{mg} / \mathrm{mL}$ MTT. The cells were incubated with MTT for 4 hours, then lysed with DMSO after removing the medium. The amount of formazan product was analyzed spectrophotometrically at an absorbance of $490 \mathrm{~nm}$.

\section{Fluorescence staining}

MitoTracker Green (MTG) staining solution was prepared and diluted to $1: 200$. After incubation with various concentrations of ALA and/or MET at $37{ }^{\circ} \mathrm{C}$ for 4 hours, cells were irradiated, then incubated for another 4 hours. The culture medium was then discarded, and the cells were washed twice with PBS, and stained (1:200) with MTG for 15 minutes. After washing twice with PBS, the cells were permeabilized with triton-100 for 10 minutes, then fixed with glycerol and sealed.

\section{Computerized simulation by CompuSyn}

Synergism or antagonism is to know both the "potency" and the "shape" of the dose-effect curve for each drug. Therefore, it is necessary to calculate the does-effect parameters of each drug alone $\left[\mathrm{m}_{1},(\mathrm{Dm})_{1}, \mathrm{~m}_{2}\right.$ and $\left.(\mathrm{Dm})_{2}\right]$ as well as in combination $\left[\mathrm{m}_{1,2},(\mathrm{Dm})_{1,2}\right]$, and thereby determine the Cmbinatioon Index (CI) value. The above parameters can be easily and automatically determined from the median-effect equation by CompuSyn software (Available for free download from www.combosyn.com). The OPDT mediated by ALA and MET was quantitatively determined for synergism [combination index $(\mathrm{CI}<1)$, antagonism $(\mathrm{CI}>1)$ and additive effect $(\mathrm{CI}=1)]$ in vitro. The theoretical algorithm and the CompuSyn software simulation have been used to illustrate step-by-step for the combination of two anti-cancer agents.

\section{Statistical analysis}

All experiments were repeated at least three times with six parallel wells. The resulting values were expressed as the relative number of cells per well when compared to the control groups. The results were averaged for experiments performed under similar conditions and were expressed as mean $\pm \mathrm{SD}$.

\section{Results}

OPDT and PDT effects on MDA-MB-231 cell viability at different radiation energies

To investigate the effects of OPDT and PDT using different light radiation energy intensities, MDA-MB-231 cells were incubated with $1 \mathrm{mM}$ ALA for 4 hours. Cells were exposed to different energy intensities of the OPDT and PDT light sources. Cell survival was analyzed using an MTT assay 72 hours after irradiation. The results showed that with increasing irradiation intensity $(0,2,6,12,24$, and $\left.36 \mathrm{~J} / \mathrm{cm}^{2}\right)$, the cell survival rates gradually decreased (Figure 1). OPDT resulted in slightly lower cell viability than PDT. $T$-test analysis of the cell survival rate under different energy intensities showed no significant differences 


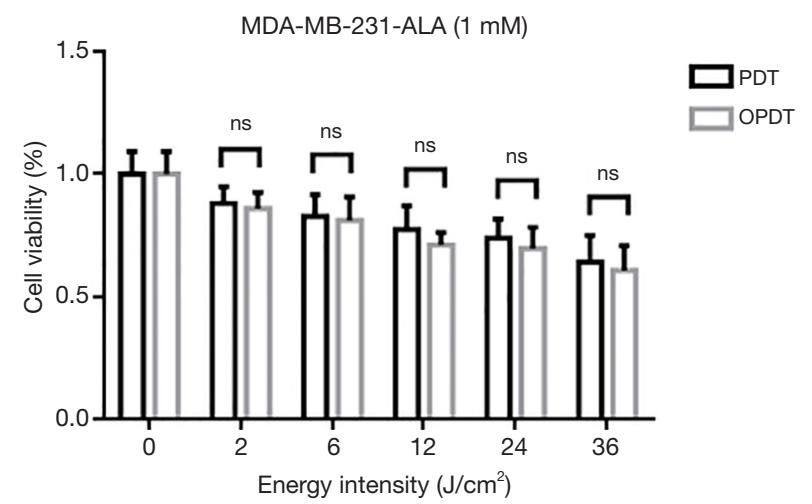

Figure 1 Cell viability after irradiation of different energy intensities. MDA-MB-231 cells were incubated with $1 \mathrm{mM}$ 5-aminolevulinic acid (ALA) and irradiated under different energy intensities $\left(0,2,6,12,24\right.$, and $\left.36 \mathrm{~J} / \mathrm{cm}^{2}\right)$ with a diode laser with a $633 \mathrm{~nm}$ wavelength emission. T-test analysis was performed between the two groups at the same energy intensity (ns, no statistical difference; $\mathrm{P}>0.05, \mathrm{n}=4)$.

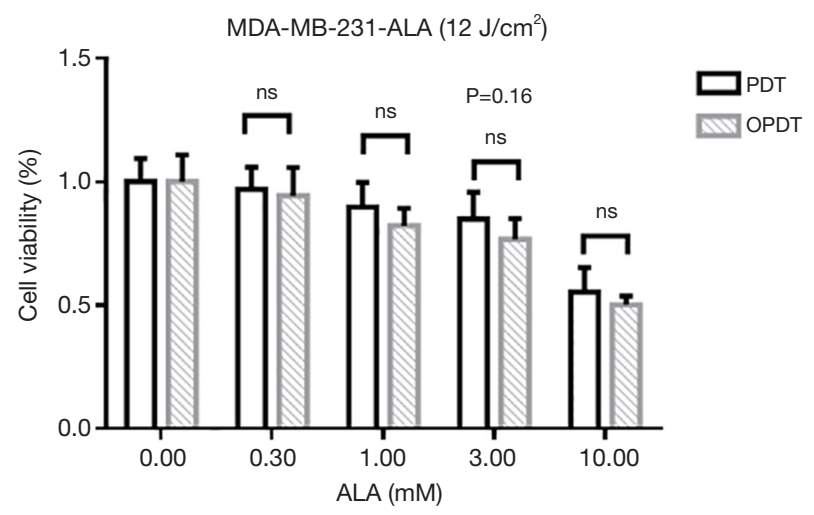

Figure 2 Effects of photodynamic therapy (PDT) and oxyphotodynamic therapy (OPDT) on cell survival after incubation with different concentrations of 5-aminolevulinic acid (ALA). MDAMB-231 cells were incubated with different concentrations of ALA $(0,0.3,1,3$, and $10 \mathrm{mM})$, then treated with OPDT/PDT $\left(12 \mathrm{~J} / \mathrm{cm}^{2}\right)$. $T$-test analysis was performed between the two groups at the same concentration (ns, no statistical difference; $\mathrm{P}>0.05, \mathrm{n}=4$ ).

between each group, with their respective $\mathrm{P}$ values of $0,0.82$, $0.77,0.55,0.47$, and 0.22 .

\section{OPDT and PDT effects on MDA-MB-231 cell viability in combination with different concentrations of ALA}

MDA-MB-231 cells were incubated with different concentrations of ALA for 4 hours, then treated with OPDT or PDT $\left(12 \mathrm{~J} / \mathrm{cm}^{2}\right)$. Cell survival was analyzed using an MTT assay 72 hours after irradiation. The results showed that with the increasing concentrations of ALA, cell survival gradually decreased (Figure 2). OPDT resulted in slightly lower cell viability than PDT; however, no statistical differences existed between each group, with respective $\mathrm{P}$ values of $0,0.99,0.81,0.16$, and 0.81 .

\section{Effects of ALA-mediated OPDT and PDT on MDA- $M B-231$ cell viability}

MDA-MB-231 cells were seeded in 96-well plates at a density of 7500 cells/well in $150 \mu \mathrm{L}$ DMEM medium. After reaching $70 \%$ confluence, the cells were incubated with different concentrations of ALA (0, 0.25, 0.5, 1, 2, 4, 8, 16, 24 , and $32 \mathrm{mM}$ ) for 72 hours. The cells were divided into three groups: Group 1 (ALA), Group 2 (ALA-PDT), and Group 3 (ALA-OPDT), as shown in Figure $3 A, B, C$. The $\mathrm{IC}_{50}$ value of each group was $14.03 \pm 1.10,10.62 \pm 1.22$, and $7.71 \pm 1.26 \mathrm{mM}$, respectively (Figure $3 D$ ).

\section{Effects of MET-mediated OPDT and PDT on MDA- MB-231 cell viability}

MDA-MB-231 cells were seeded in 96-well plates at a density of 7,500 cells/well in $150 \mu \mathrm{L}$ DMEM medium. After reaching $70 \%$ confluence, the cells were incubated with different concentrations of MET $(0,0.25,0.5,1,2,4$, $8,16,24$, and $32 \mathrm{mM}$ ) for 72 hours. The cells were divided into three groups: Groups 1 (MET), Group 2 (METPDT), and Group 3 (MET-OPDT) (Figure $4 A, B, C$ ). The $\mathrm{IC}_{50}$ value of each group was $18.27 \pm 1.04,22.09 \pm 1.05$, and $23.96 \pm 1.05 \mathrm{mM}$, respectively (Figure $4 D$ ).

\section{Cell survival rate analysis of ALA and MET combined with OPDT and PDT}

MDA-MB-231 cells were seeded in 96-well plates at a density of 7,500 cells/well in $150 \mu \mathrm{L}$ DMEM medium. After reaching $70 \%$ confluence, the cells were incubated with different concentrations of both ALA and MET (0, 0.25, $0.5,1,2,4,8,16,24$, and $32 \mathrm{mM}$ ) for 72 hours. The cells were then divided into 3 groups: Group 1 (ALA-MET), Group 2 (ALA-MET-PDT), and Group 3 (ALA-METOPDT). The concentration ratio of ALA and MET was 1:1. Concentrations of 24 and $32 \mathrm{mM}$ resulted in cell death (Figure $5 A, B, C$ ). 

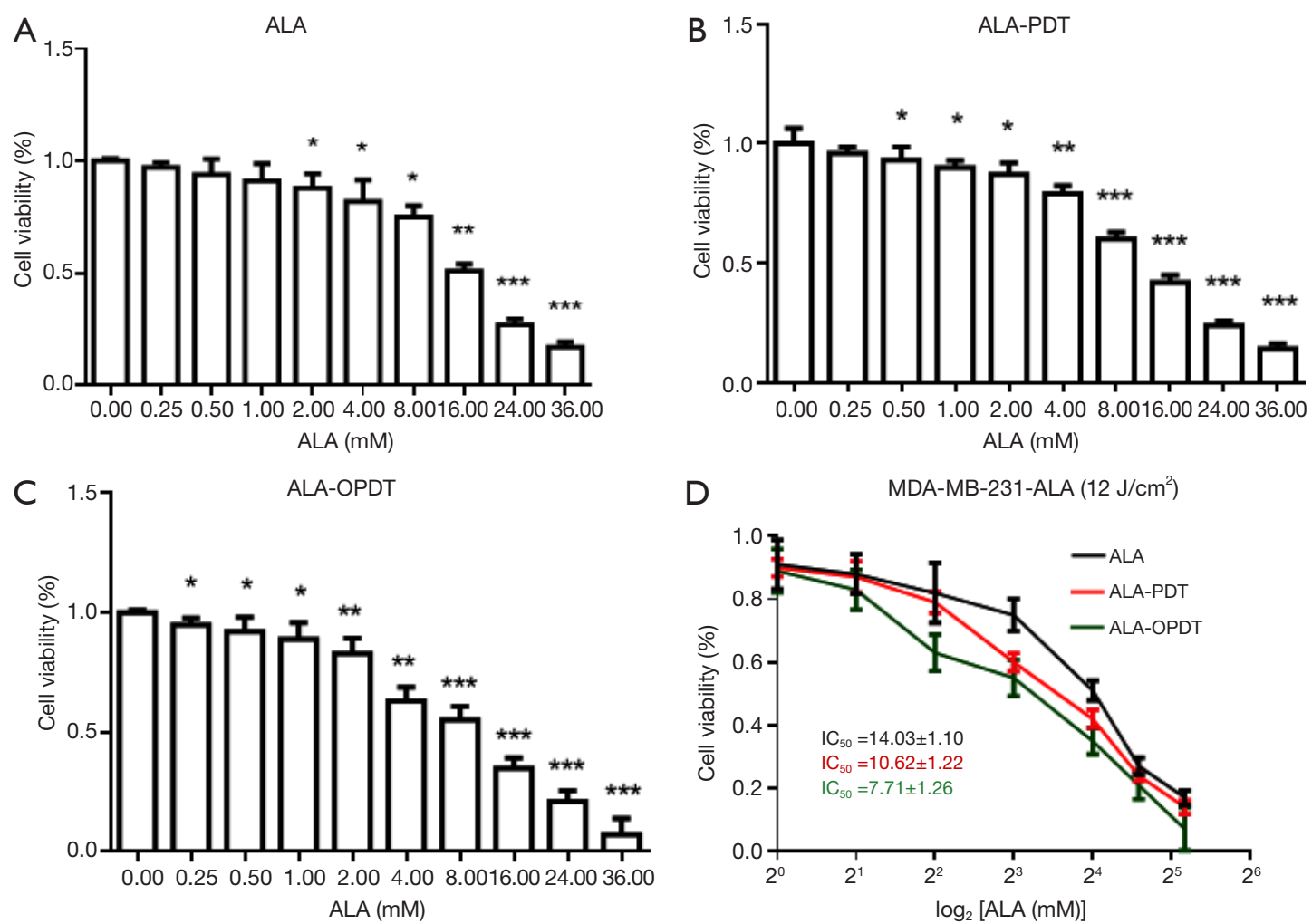

Figure 3 Cell viability of MDA-MB-231 cells following photodynamic therapy (PDT) or oxyphotodynamic therapy (OPDT) and incubation with different 5-aminolevulinic acid (ALA) concentrations. (A) Inhibitory effects of ALA on MDA-MB-231 cells at different concentrations; (B) inhibitory effects of ALA-PDT on MDA-MB-231 cells at different concentrations; (C) inhibitory effects of ALA-OPDT on MDAMB-231 cells at different concentrations; (D) cell survival rates in the mentioned 3 groups at different concentrations of ALA. Each group was compared with the control group $(0 \mathrm{mM})$. *, $\mathrm{P}<0.05 ;{ }^{* *}, \mathrm{P}<0.01$; ${ }^{* * *}, \mathrm{P}<0.001$.

\section{Drug concentration design and computerized simulation by CompuSyn}

To determine the synergism or antagonism of the drug combination of ALA and MET in OPDT/PDT, the median-effect equation using CompuSyn software was utilized. Dose-effect parameters of each drug alone $\left[\mathrm{m}_{1}\right.$, $(\mathrm{Dm})_{1}, \mathrm{~m}_{2}$ and $\left.(\mathrm{Dm})_{2}\right]$ as well as in combination $\left[\mathrm{m}_{1,2}\right.$, $\left.(\mathrm{Dm})_{1,2}\right]$ were calculated to determine the CI value (22-24). The ratio of the combination of the two drugs depends on the ratio of $\mathrm{IC}_{50}$ values. In this study, the $\mathrm{IC}_{50}$ values of ALA and MET were $14.03 \pm 1.10$ and $18.27 \pm 1.04 \mathrm{mM}$, respectively. Therefore, combination therapy was carried out at a ratio of $1: 1$. Concomitantly, the combined effect of the two drugs was compared after OPDT and PDT. Under different conditions (control, PDT, and OPDT), the effect of ALA combined with MET was significantly different. In the absence of light, ALA and MET played a synergistic role (Table 1 and Figures S1 and 6), with $\mathrm{CI}<1$. In the OPDT and PDT groups, ALA and MET played a synergistic role (Table 1 and Figures $S 2, S 3$ and 7,8).

\section{OPDT and PDT induced mitochondrial phototoxicity}

To determine the effect of ALA-PDT and ALA-OPDT with or without MET, MDA-MB-231 cells were stained with MTG (1:200) for 4 hours following PDT or OPDT. The fluorescence of PpIX and MTG was obtained by different excitation wavelengths under a confocal microscope. Faint fluorescence of PpIX was observed in the ALA ( $2 \mathrm{mM}$ ) group (Figure $9 A, B, C)$, and ALA-MET $(2 \mathrm{mM})$ group (Figure $9 D, E, F)$, with no significant increases. However, when OPDT or PDT (200 J/ $\mathrm{cm}^{2}$ fluence) were combined with ALA and MET, the fluorescence intensity of PpIX was significantly enhanced, and OPDT had the greatest effect (Figure 9G,H,I,7,K,L). 

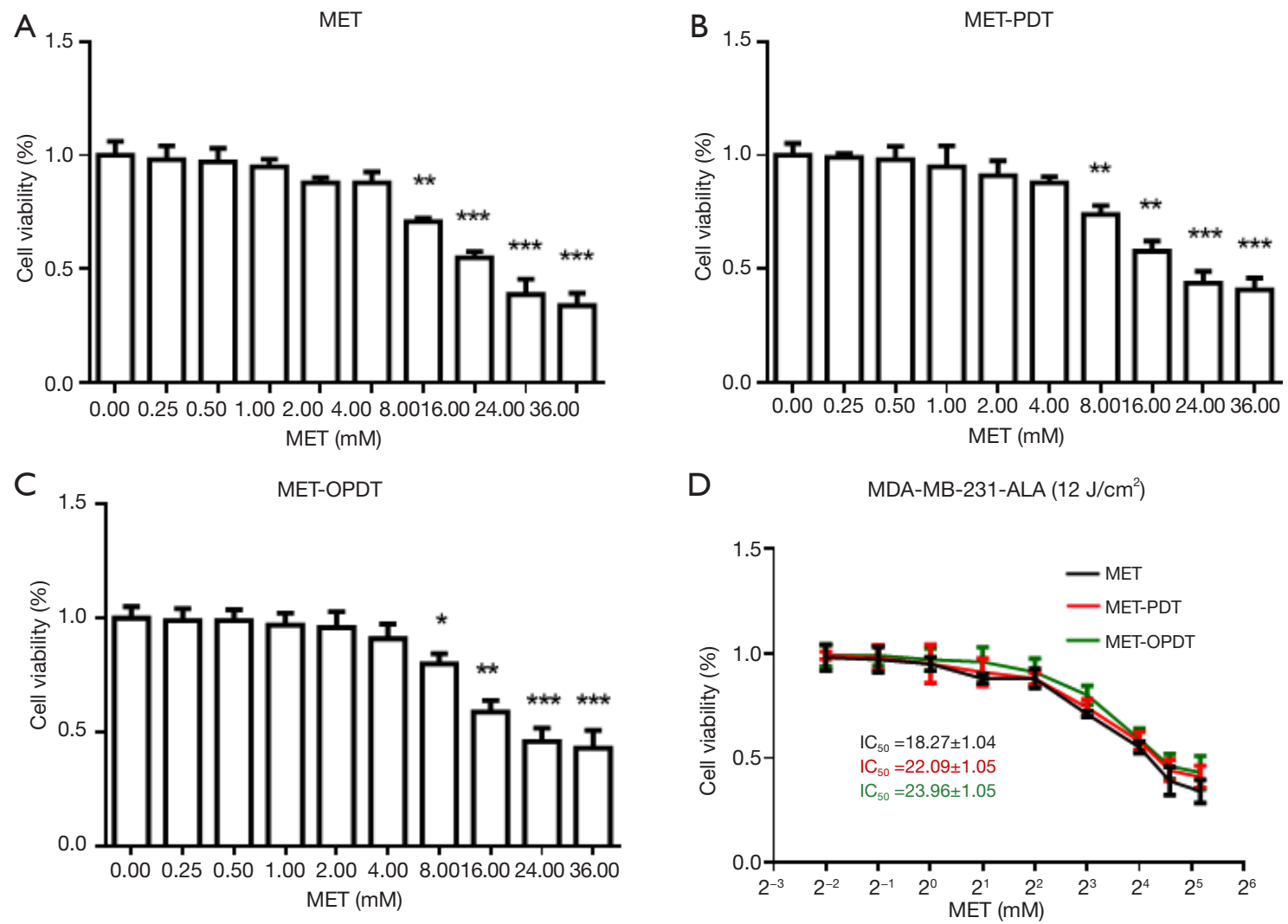

Figure 4 Cell viability of MDA-MB-231 cells following photodynamic therapy (PDT) or oxyphotodynamic therapy (OPDT) and incubation with different concentrations of metformin (MET). (A) Inhibitory effects of MET on MDA-MB-231 cells at different concentrations; (B) inhibitory effects of MET- PDT on MDA-MB-231 cells at different concentrations; (C) inhibitory effects of MET-OPDT on MDAMB-231 cells at different concentrations; (D) cell survival rates in the mentioned 3 groups at different concentrations of MET. Each group was compared with the control group $(0 \mathrm{mM})$. *, $\mathrm{P}<0.05$; ${ }^{* *}, \mathrm{P}<0.01$; ***, $\mathrm{P}<0.001$.

\section{Discussion}

PDT is a novel method for treating breast cancer (21). In PDT, photosensitizers can degrade intracellular oxygen more rapidly under laser irradiation, keeping cells in a hypoxic state (25). However, OPDT may alleviate the above problems. In this study, pure oxygen was transported through a specially designed gas, light, and electricity irradiator using novel oxygen production technology. The three factors do not interfere with each other, and oxygen and the irradiation light source synergistically act in the target area. The problem of tumor hypoxia can be overcome by using OPDT equipment and optimized photosensitizing drug concentrations, thereby improving breast cancer treatment.

As shown in Figure 10, ALA generates PpIX through a series of reactions. Excitation of PpIX generates a large number of singlet oxygen and free radicals. In the absence of light, PpIX and ferrous ions form heme, catalyzed by iron chelating enzymes. Heme is decomposed into NO, iron and biliverdin through HO-1 catalysis. Heme interacts with MET to exert a synergistic effect and regulate the apoptosis and proliferation of cancer cells (14). As an upstream product of heme, ALA combined with MET to induce the synergistic effects demonstrated in the present study. According to the CompuSyn software $(26,27)$, the ratio of combination of ALA and MET was calculated to be the ratio of the $\mathrm{IC}_{50}$ values of the two drugs, and the concentration remained as diluted as possible. The inhibitory rate of the ALA group gradually increased with increasing doses. When the concentration reached $2 \mathrm{mM}$, the inhibitory rate of the ALA group was significantly higher than that of the control group, indicating that ALA alone had an anti-tumor effect. The $\mathrm{IC}_{50}$ values of the two drug groups also confirmed that for OPDT, the oxygen supply improves the hypoxic state of 


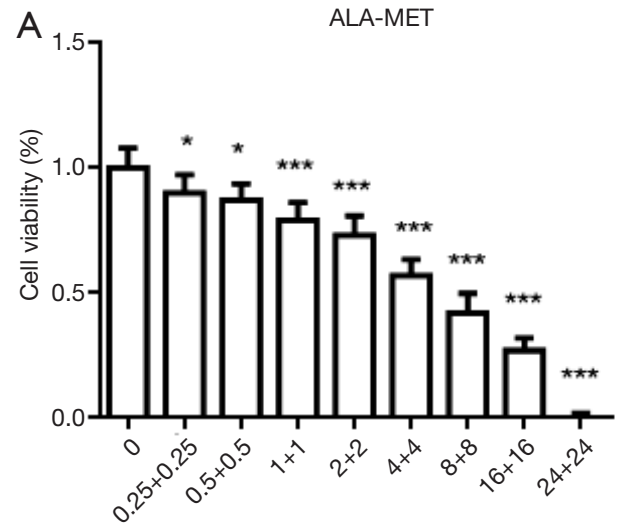

ALA-MET (mM)

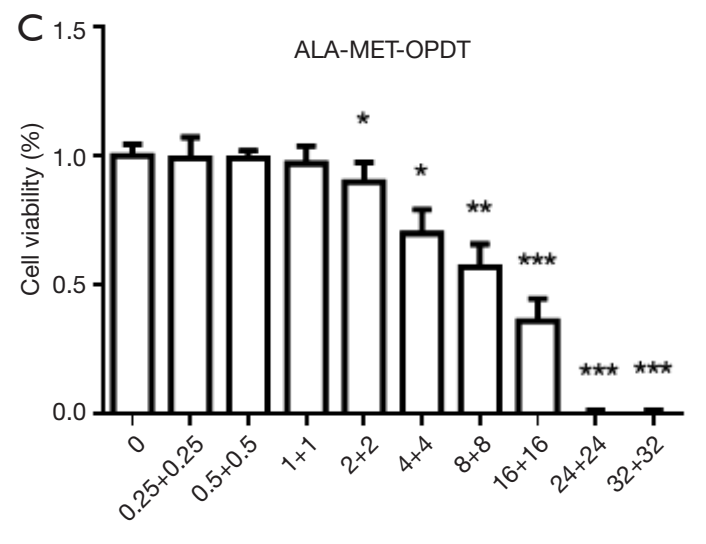

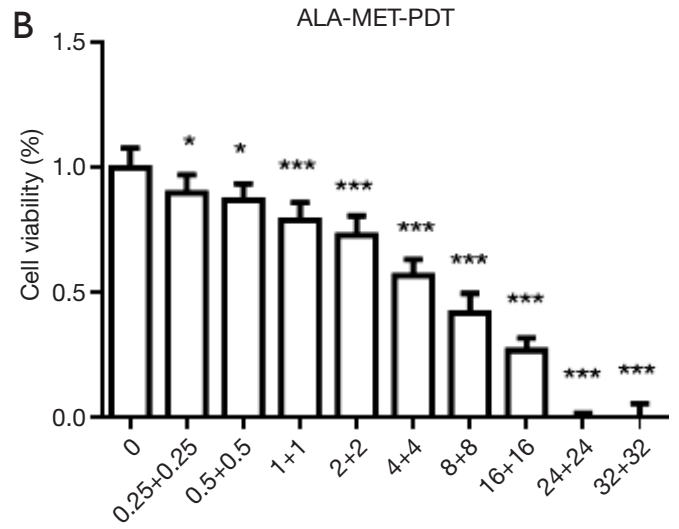

ALA-MET (mM)

ALA-MET (mM)

Figure 5 Cell viability of MDA-MB-231 cells following photodynamic therapy (PDT) or oxyphotodynamic therapy (OPDT) and incubation with different concentrations of combined ALA and MET for 72 hours, measured with the MTT assay. (A) ALA-MET; (B) ALA-METPDT; (C) ALA-MET-OPDT. Each group was compared with the control group $(0 \mathrm{mM}) .{ }^{*}, \mathrm{P}<0.05,{ }^{* *}, \mathrm{P}<0.01,{ }^{* * *}, \mathrm{P}<0.001$.

Table 1 Comparison of drug combinations for OPDT or PDT against MDA-MB-231 cell growth in MTT assay in vitro

\begin{tabular}{lccc}
\hline $\mathrm{Cl}$ & ALA + MET & $\begin{array}{c}\text { ALA + MET + } \\
\text { PDT }\end{array}$ & ALA + MET + OPDT \\
\hline $\mathrm{ED}_{50}$ & 0.30384 & 0.47467 & 0.34607 \\
$\mathrm{ED}_{75}$ & 0.13569 & 0.19790 & 0.08585 \\
$\mathrm{ED}_{90}$ & 0.06086 & 0.08254 & 0.02203 \\
$\mathrm{ED}_{95}$ & 0.03537 & 0.04554 & 0.00891 \\
Conclusion & Synergism & Synergism & Synergism \\
\hline
\end{tabular}

$\mathrm{Cl}$ data can be calculated by the CompuSyn soft, $\mathrm{Cl}<1, \mathrm{Cl}$ $>1$ and $\mathrm{Cl}=1$ indicate synergism, antagonism and additivity, respectively. ED: Maximum reaction intensity dosage.

the cells, increasing the production of oxygen free radicals or singlet oxygen, thereby decreasing cancer cell viability.
Under confocal laser microscopy, the fluorescence intensity of PpIX under OPDT was significantly stronger than that of traditional PDT.

MET is a classical hypoglycemic drug. In addition to its hypoglycemic mechanism of action, it also has anti-tumor effects via the AMPK signaling pathway (17). However, the anticancer potential of MET in its conventional therapeutic dosage is extremely small. Previous studies have confirmed that the concentration of MET that has the greatest anticancer potential in preclinical models is within the range of $\mathrm{mM}$ (28), which is higher than that of serum MET with conventional anti-diabetic dosage. Therefore, for PDTresistant tumors, combination therapy with MET should be used for maximal therapeutic efficacy.

The results of this study showed no significant difference in the cell survival rate between laser irradiation and non-laser irradiation. Even in the PDT and OPDT 

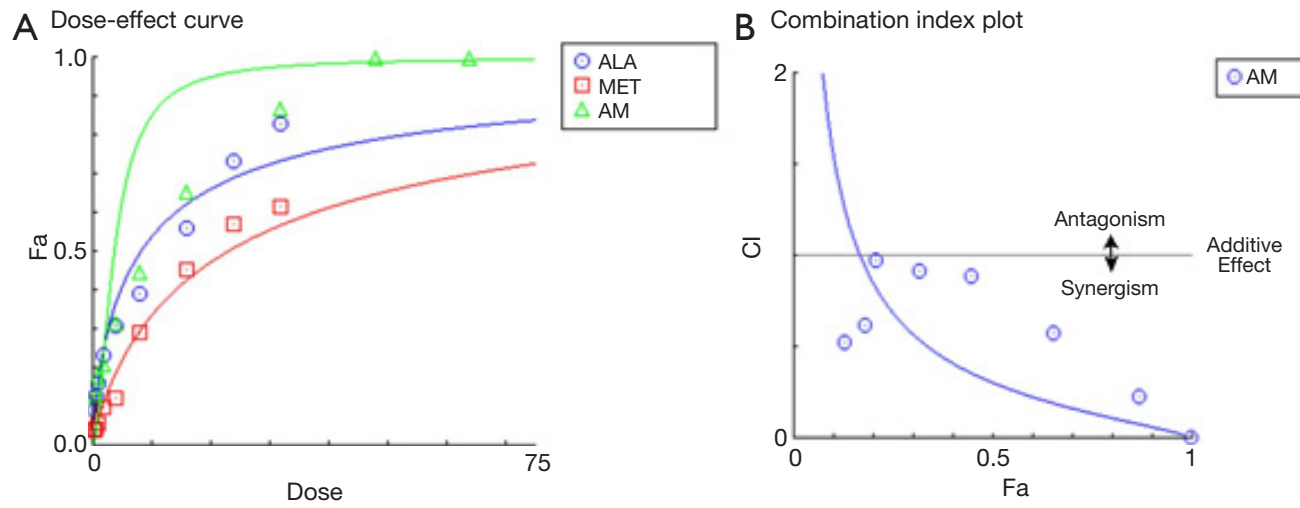

Figure 6 Graphical presentations obtained from the CompuSyn Report for the ALA and MET combinations. (A) Dose-effect curves; (B) median-effect plot. AM, ALA-MET.
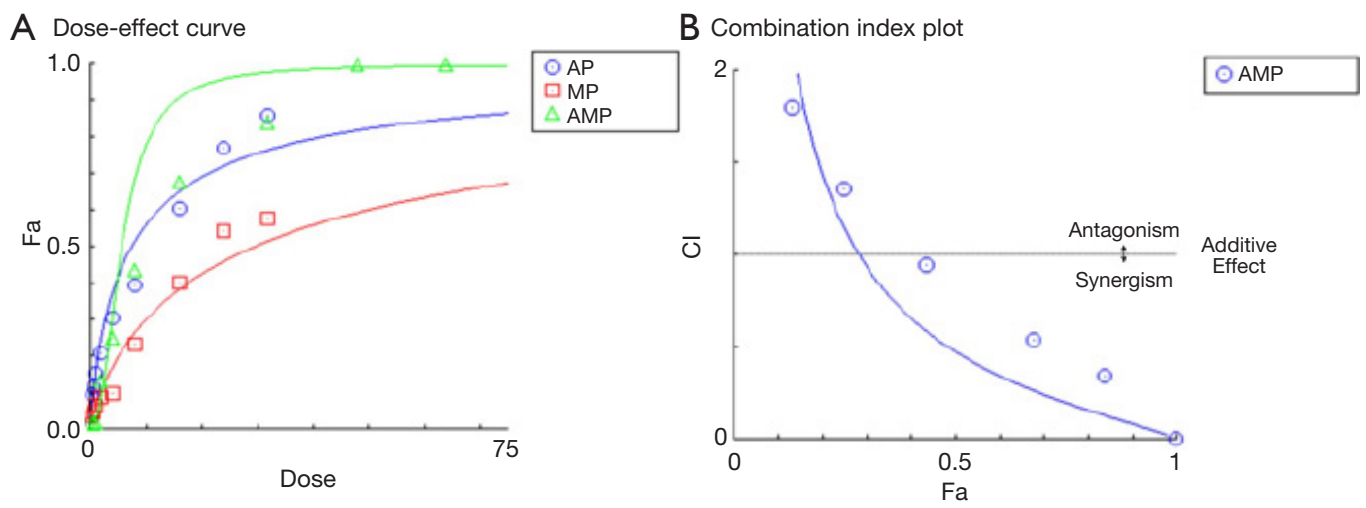

Figure 7 Graphical presentations obtained from the CompuSyn Report for the ALA-PDT and MET-PDT combinations (A) Dose-effect curves; (B) median-effect plot. AMPP, ALA-MET-PDT.
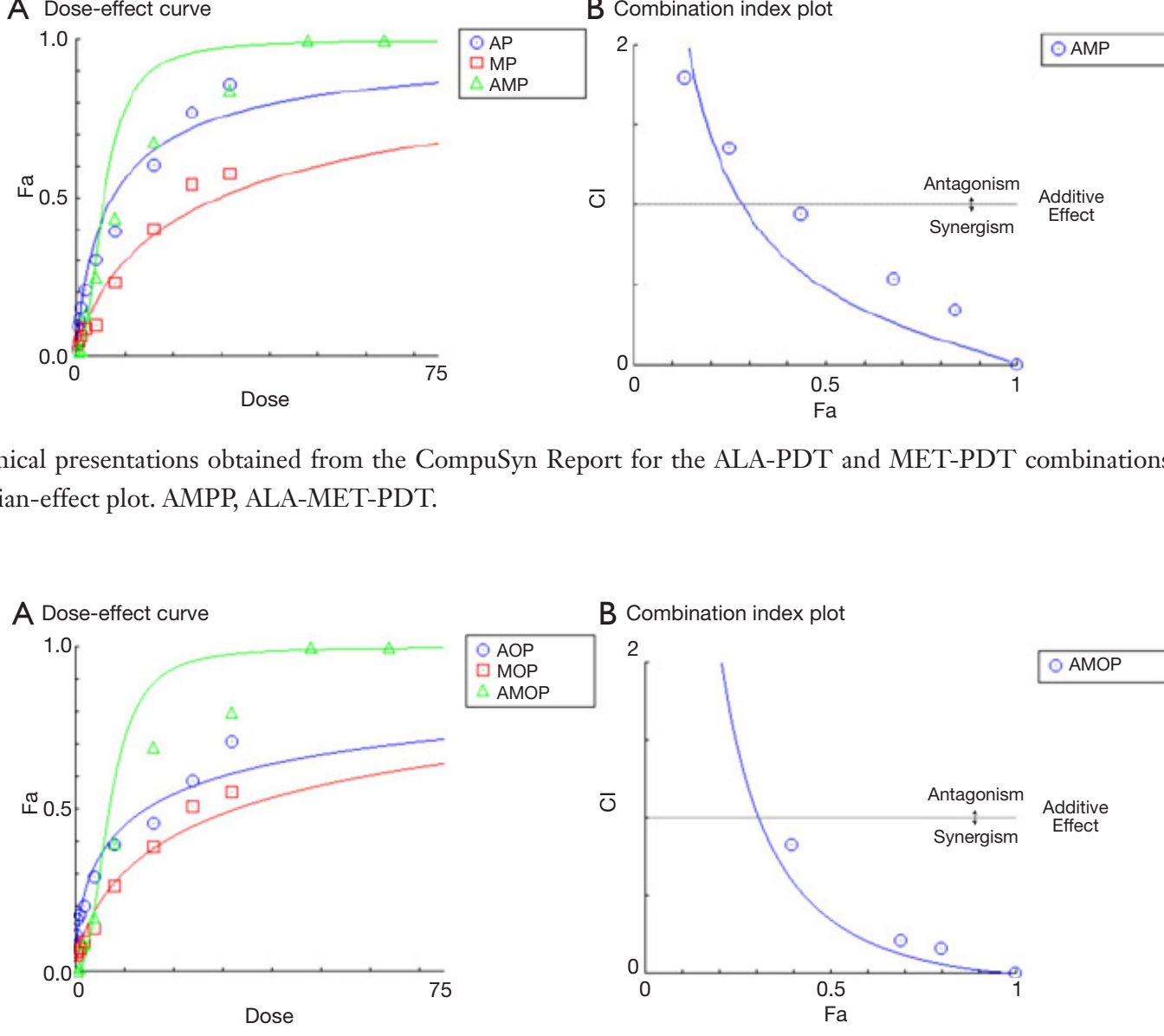

Figure 8 Graphical presentations obtained from the CompuSyn Report for the ALA-OPDT and MET-OPDT combinations. (A) Doseeffect curves; (B) median-effect plot. AMOP, ALA-MET-OPDT. 


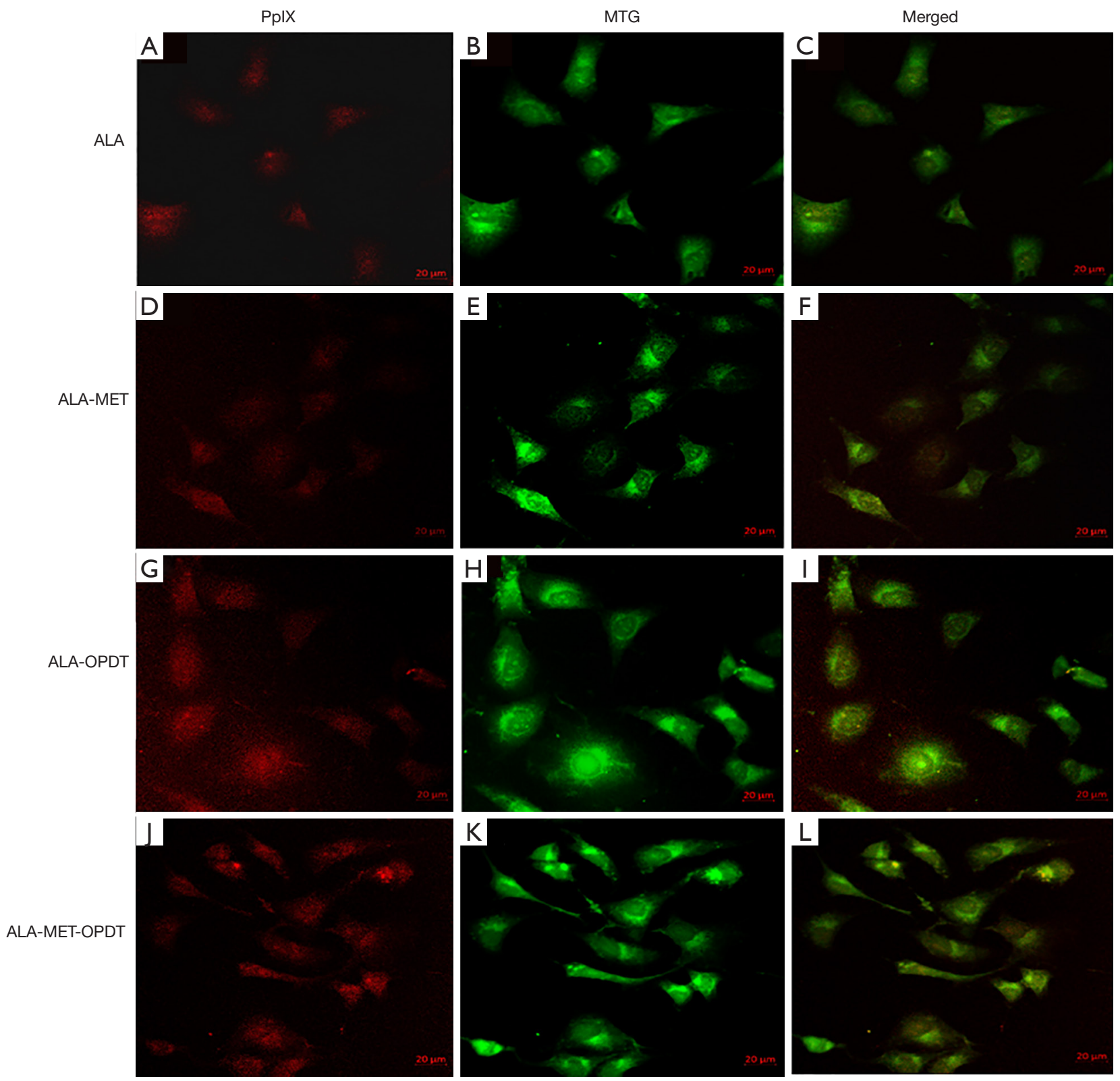

Figure 9 Confocal microscopy analysis of (A,D,G,J) Protoporphyrin IX (PpIX) and (B,E,H,K) MitoTracker Green (MTG) fluorescence in MDA-MB-231 cells after oxyphotodynamic therapy (OPDT). Cells were incubated with $2 \mathrm{mM}$ ALA then irradiated at $12 \mathrm{~J} / \mathrm{cm}^{2}$. (C,F,I,L) Co-localization of mitochondria. Fluorescence images were captured through the control of a monochromator under the conditions of $610 \pm 20 \mathrm{~nm}$ for PpIX and 530 $\pm 30 \mathrm{~nm}$ for MTG.

groups, laser irradiation stimulates the release of certain cytokines, causing reparative effects on the damaged cells. Oxygen supply promotes the energy metabolism of mitochondria (15). When exogenous ALA enters the cells, heme synthesis increases, and the synergistic effect of ALA and MET significantly increases. PDT and PpIX induce the generation of a large amount of singlet oxygen and reactive oxygen species, causing the cells to enter a hypoxic state. During ALA-OPDT treatment, continuous external oxygen production effectively alleviates the 

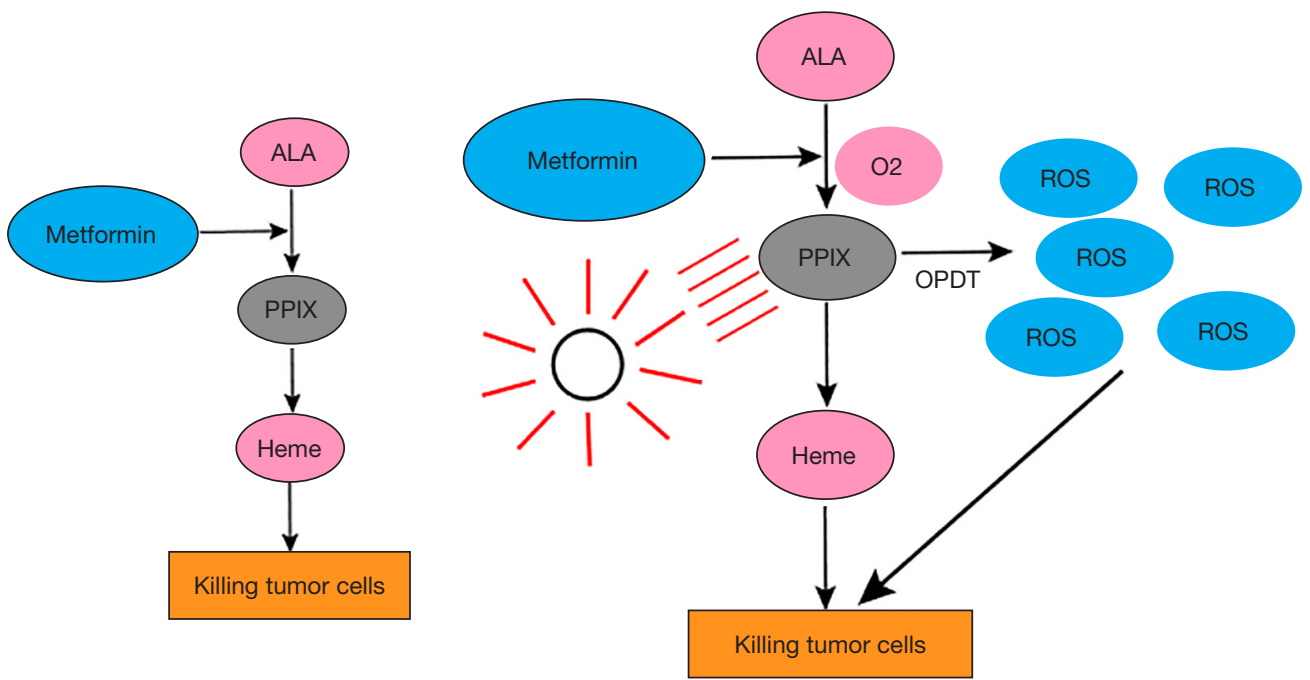

Figure 10 Metformin (MET) combined with hemoglobin synthesized by exogenous ALA acts on tumor cells. Under the intervention of oxyphotodynamic therapy (OPDT), more ROS is generated and combined with hemoglobin to kill tumor cells.

hypoxic state in cells. However, for both ALA-PDT or ALA-OPDT, exogenous ALA enters the mitochondria. Under laser irradiation, more PpIX decomposes into reactive oxygen species, induces apoptosis and necrosis, reduces heme synthesis, and reduces the synergistic effect of ALA and MET. MET also protects cells from hydrogen peroxide damage through the AMPK/C/EBP B/MIR-1A3P/GRP94 signaling pathway, and inhibits the production of reactive oxygen species, reducing the killing effect of PDT/OPDT on cancer cells $(29,30)$. Therefore, the fluorescence expression of the ALA-MET-PDT and ALAMET-OPDT group was significantly stronger than that of the combination group. As shown in Figure $5 A, B$, the combination of ALA-MET-PDT and ALA-MET-OPDT showed a synergistic effect. The CompuSyn software was used to calculate the CI, which was $<1$. A low dose of reactive oxygen species can activate the Wnt signaling transduction pathway and promote cell proliferation and differentiation. This was evident in MDA-MB-231 cells, which were not sensitive to ALA-PDT.

In summary, this study introduces OPDT as a novel antitumor therapy for TBNC. It has a similar mode of action to PDT, but additionally, it solves the problem of tumor hypoxia by generating external oxygen. The mechanism of the therapeutic effect of OPDT is summarized as follows: (I) ROS are produced, especially singlet oxygen, which can directly kill the tumor cells (induce apoptosis or necrosis); (II) OPDT directly activates the body's anti-tumor immune response; and (III) OPDT damages the vascular system surrounding the tumor, leading to ischemic tumor shrinkage. This study also demonstrated that the combined use of ALA and MET had a synergistic anti-tumor effect in TNBC cells when combined with OPDT. Further studies that elucidate the underlying cellular and molecular mechanisms of OPDT in treating tumors are warranted.

\section{Acknowledgments}

Funding: This work was funded by the National Key R\&D Program of China (2018YFC0910601), the National Natural Science Foundation of China (No. 81871382, 81870019), Key Realm R\&D Program of Guangdong Province 2018B030337001, and Starting Fund from Sun Yat-sen University Fifth Affiliated Hospital.

\section{Footnote}

Reporting Checklist: The authors have completed the MDAR reporting checklist. Available at http://dx.doi.org/10.21037/ atm-20-5704

Data Sharing Statement: Available at http://dx.doi. org/10.21037/atm-20-5704

Conflicts of Interest: All authors have completed the ICMJE uniform disclosure form (available at http://dx.doi. org/10.21037/atm-20-5704). The authors have no conflicts of interest to declare. 
Ethical Statement: The authors are accountable for all aspects of the work in ensuring that questions related to the accuracy or integrity of any part of the work are appropriately investigated and resolved.

Open Access Statement: This is an Open Access article distributed in accordance with the Creative Commons Attribution-NonCommercial-NoDerivs 4.0 International License (CC BY-NC-ND 4.0), which permits the noncommercial replication and distribution of the article with the strict proviso that no changes or edits are made and the original work is properly cited (including links to both the formal publication through the relevant DOI and the license). See: https://creativecommons.org/licenses/by-nc-nd/4.0/.

\section{References}

1. Ackroyd R, Kelty C, Brown N, et al. The history of photodetection and photodynamic therapy. Photochem Photobiol 2001;74:656-69.

2. Maehara S, Ikeda N. Photodynamic Therapy. Kyobu Geka 2018;71:858-61.

3. Dang J, He H, Chen D, et al. Manipulating tumor hypoxia toward enhanced photodynamic therapy (PDT). Biomater Sci 2017;5:1500-11.

4. Rkein AM, Ozog DM. Photodynamic therapy. Dermatol Clin 2014;32:415-25, $\mathrm{x}$.

5. Bansal A, Yang F, Xi T, et al. In vivo wireless photonic photodynamic therapy. Proc Natl Acad Sci U S A 2018;115:1469-74.

6. Abrahamse H, Kruger CA, Kadanyo S, et al. Nanoparticles for advanced photodynamic therapy of cancer. Photomed Laser Surg 2017;35:581-8.

7. Shepherd RW, Radchenko C. Bronchoscopic ablation techniques in the management of lung cancer. Ann Transl Med 2019;7:362.

8. Kim J, Cho HR, Jeon H, et al. Continuous O2-Evolving $\mathrm{MnFe} 2 \mathrm{O} 4$ Nanoparticle-Anchored Mesoporous Silica Nanoparticles for Efficient Photodynamic Therapy in Hypoxic Cancer. J Am Chem Soc 2017;139:10992-5.

9. Liang H, Zhou Z, Luo R, et al. Tumor-specific activated photodynamic therapy with an oxidation-regulated strategy for enhancing anti-tumor efficacy. Theranostics 2018;8:5059-71.

10. Beaudoin S, Leyton JV: Development of a Novel Covalent Folate-Albumin-Photosensitizer Conjugate. Photochem Photobiol 2016;92:512-4.

11. Zhu X, Wang H, Zheng L, et al. Upconversion nanoparticle-mediated photodynamic therapy induces THP-1 macrophage apoptosis via ROS bursts and activation of the mitochondrial caspase pathway. Int $\mathrm{J}$ Nanomedicine 2015;10:3719-36.

12. Wang X, Cao P, Liu J, et al. 5-Aminolaevulinic AcidBased Photodynamic Therapy Restrains Pathological Hyperplasia of Fibroblasts. Med Sci Monit 2017;23:46-56.

13. Sil S, Bose T, Roy D, Chakraborti AS. Protoporphyrin IX-induced structural and functional changes in human red blood cells, haemoglobin and myoglobin. J Biosci 2004;29:281-91.

14. Lee J, Yesilkanal AE, Wynne JP, et al. Effective breast cancer combination therapy targeting BACH1 and mitochondrial metabolism. Nature 2019;568:254-8.

15. Gao ZY, Liu Z, Bi MH, et al. Metformin induces apoptosis via a mitochondria-mediated pathway in human breast cancer cells in vitro. Exp Ther Med 2016;11:1700-6.

16. Aljofan M, Riethmacher D. Anticancer activity of metformin: a systematic review of the literature. Future Sci OA 2019;5:FSO410.

17. Howell JJ, Hellberg K, Turner M, et al. Metformin Inhibits Hepatic mTORC1 Signaling via Dose-Dependent Mechanisms Involving AMPK and the TSC Complex. Cell Metab 2017;25:463-71.

18. Jara JA, López-Muñoz R. Metformin and cancer: Between the bioenergetic disturbances and the antifolate activity. Pharmacol Res 2015;101:102-8.

19. Orecchioni S, Reggiani F, Talarico G, et al. The biguanides metformin and phenformin inhibit angiogenesis, local and metastatic growth of breast cancer by targeting both neoplastic and microenvironment cells. Int J Cancer 2015;136:E534-44.

20. Wang RX, Xu XE, Huang L, et al. eEF2 kinase mediated autophagy as a potential therapeutic target for paclitaxelresistant triple-negative breast cancer. Ann Transl Med 2019;7:783.

21. Anand S, Yasinchak A, Bullock T, et al. A non-toxic approach for treatment of breast cancer and its metastases: capecitabine enhanced photodynamic therapy in a murine breast tumor model. J Cancer Metastasis Treat 2019;5:6.

22. Chou TC. Derivation and properties of Michaelis-Menten type and Hill type equations for reference ligands. J Theor Biol 1976;59:253-76.

23. Chou TC. Synergy determination issues. J Virol 2002;76:10577; author reply 10578.

24. Chou TC, Nadas A, Zolla-Pazner S. Synergy Determination Issues. J Virol 2002;76:10577-8.

25. Varol M. Photodynamic Therapy Assay. Methods Mol Biol 
Page 12 of 12

2020;2109:241-50.

26. Chou TC. The combination index $(\mathrm{CI}<1)$ as the definition of synergism and of synergy claims. Synergy 2018;7:49-50.

27. Chou TC. The mass-action law based algorithm for cost-effective approach for cancer drug discovery and development. Am J Cancer Res 2011;1:925-54.

28. Chandel NS, Avizonis D, Reczek CR, et al. Are Metformin Doses Used in Murine Cancer Models Clinically Relevant?
Cell Metab 2016;23:569-70.

29. Cheung EC, Lee P, Ceteci F, et al. Opposing effects of TIGAR- and RAC1-derived ROS on Wnt-driven proliferation in the mouse intestine. Genes Dev 2016;30:52-63.

30. Zhang Y, Liu X, Zhang L, et al. Metformin Protects against $\mathrm{H} 2 \mathrm{O} 2$-Induced Cardiomyocyte Injury by Inhibiting the miR-1a-3p/GRP94 Pathway. Mol Ther Nucleic Acids 2018;13:189-97.
Cite this article as: Pei X, Wang X, Xian J, Mi J, Gao J, Li X, Li Z, Yang M, Bi L, Yan Y, Lv W, Jin H. Metformin and oxyphotodynamic therapy as a novel treatment approach for triple-negative breast cancer. Ann Transl Med 2020;8(18):1138. doi: $10.21037 / \mathrm{atm}-20-5704$ 
Supplementary

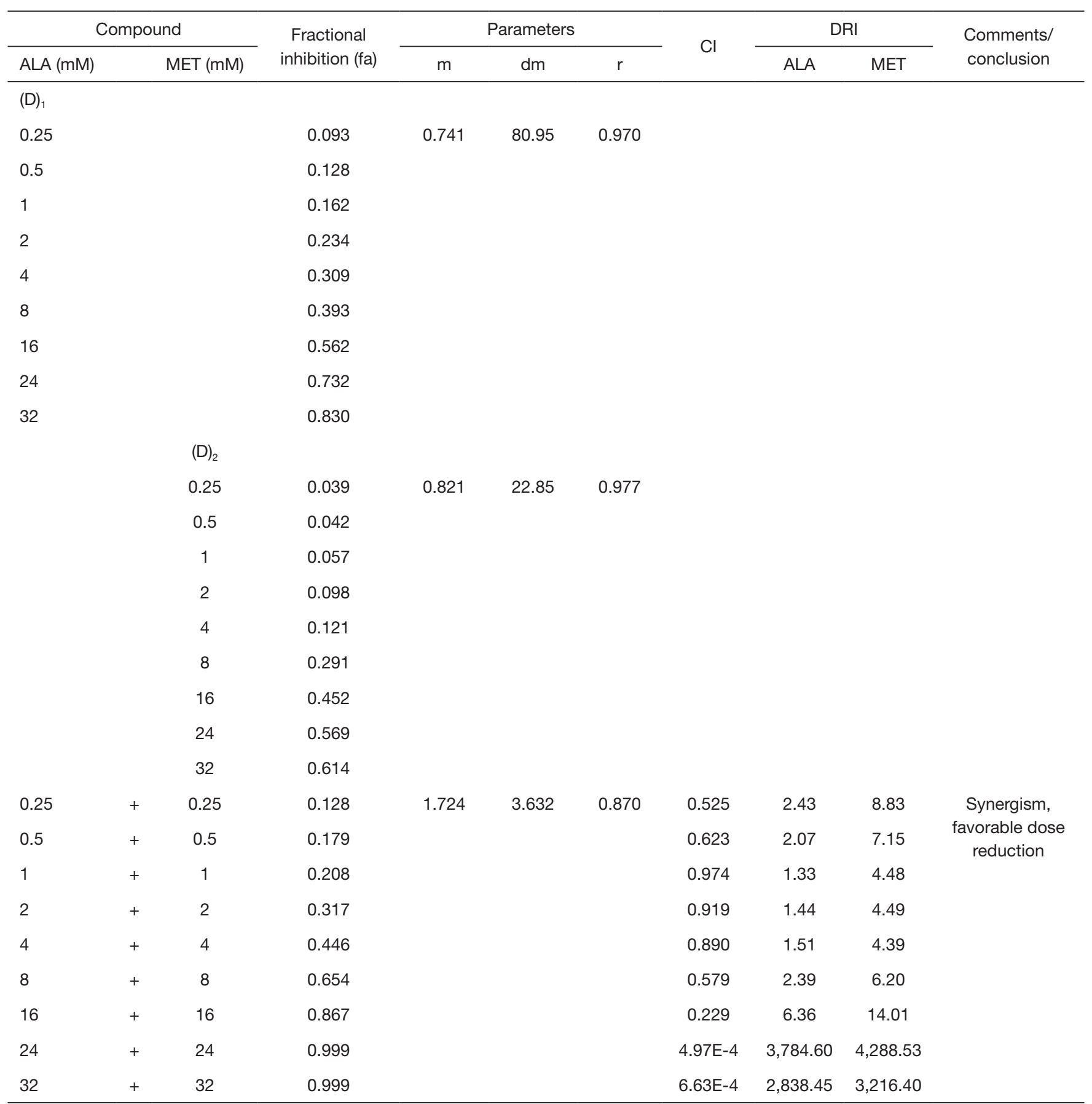

Figure S1 Combination of ALA and MET against MDA-MB-231 cell growth in MTT assay in vitro. The table is constructed by the contents generated from CompuSyn Report. (I) The dose and effect data were obtained from MTT assay (average value of triplicate) and were subjected to CompuSyn analysis. (II) Parameters were calculated from the median-effect equation and the median-effect plot. The " $m$ " is the slope, which signifies shape; $\mathrm{Dm}$ is the $\mathrm{IC}_{50}$ value (ALA in $\mathrm{mM}$, MET in $\mathrm{mM}$ ) that signifies potency; and $\mathrm{r}$ is the linear correlation coefficient that signifies conformity. (III) Combination index (CI) was calculated from the CI equation algorithms using CompuSyn software. CI $=1,<1$ and $>1$ indicates additive effect, synergism and antagonism, respectively. (IV) The dose-reduction index (DRI) was calculated from the DRI equation and algorithm using CompuSyn software. 


\begin{tabular}{|c|c|c|c|c|c|c|c|c|c|c|}
\hline \multicolumn{3}{|c|}{ Compound } & \multirow{2}{*}{ Fractional inhibition (fa) } & \multicolumn{3}{|c|}{ Parameters } & \multirow{2}{*}{$\mathrm{Cl}$} & \multicolumn{2}{|c|}{ DRI } & \multirow{2}{*}{$\begin{array}{l}\text { Comments/ } \\
\text { conclusion }\end{array}$} \\
\hline AP $(\mathrm{mM})$ & & $\mathrm{MP}(\mathrm{mM})$ & & $\mathrm{m}$ & $\mathrm{dm}$ & $r$ & & AP & MP & \\
\hline \multicolumn{11}{|l|}{ (D) $)_{1}$} \\
\hline 0.25 & & & 0.094 & 0.801 & 7.30 & 0.965 & & & & \\
\hline 0.5 & & & 0.117 & & & & & & & \\
\hline 1 & & & 0.153 & & & & & & & \\
\hline 2 & & & 0.209 & & & & & & & \\
\hline 4 & & & 0.306 & & & & & & & \\
\hline 8 & & & 0.396 & & & & & & & \\
\hline 16 & & & 0.605 & & & & & & & \\
\hline 24 & & & 0.770 & & & & & & & \\
\hline \multirow[t]{11}{*}{32} & & & 0.859 & & & & & & & \\
\hline & & $(\mathrm{D})_{2}$ & & & & & & & & \\
\hline & & 0.25 & 0.035 & 0.775 & 29.36 & 0.971 & & & & \\
\hline & & 0.5 & 0.046 & & & & & & & \\
\hline & & 1 & 0.065 & & & & & & & \\
\hline & & 2 & 0.089 & & & & & & & \\
\hline & & 4 & 0.099 & & & & & & & \\
\hline & & 8 & 0.233 & & & & & & & \\
\hline & & 16 & 0.233 & & & & & & & \\
\hline & & 24 & 0.543 & & & & & & & \\
\hline & & 32 & 0.576 & & & & & & & \\
\hline 0.25 & + & 0.25 & 0.017 & 2.178 & 5.55 & 0.932 & 6.996 & 0.185 & 0.624 & \multirow{9}{*}{$\begin{array}{l}\text { synergism } \\
\text { favorable dose- } \\
\text { reduction }\end{array}$} \\
\hline 0.5 & + & 0.5 & 0.020 & & & & 11.36 & 0.114 & 0.386 & \\
\hline 1 & + & 1 & 0.133 & & & & 1.801 & 0.705 & 2.611 & \\
\hline 2 & + & 2 & 0.250 & & & & 1.359 & 0.928 & 3.556 & \\
\hline 4 & + & 4 & 0.436 & & & & 0.945 & 1.324 & 5.266 & \\
\hline 8 & + & 8 & 0.678 & & & & 0.537 & 2.309 & 9.597 & \\
\hline 16 & + & 16 & 0.839 & & & & 0.344 & 3.574 & 2.679 & \\
\hline 24 & + & 24 & 0.999 & & & & 7.07E-4 & $1,673.02$ & $9,110.27$ & \\
\hline 32 & + & 32 & 0.999 & & & & $9.43 \mathrm{E}-4$ & $1,254.76$ & $6,832.70$ & \\
\hline
\end{tabular}

Figure S2 Combination of ALA-PDT (AP) and MET-PDT (MP) against MDA-MB-231 cell growth in MTT assay in vitro. The table is constructed by the contents generated from CompuSyn Report. (I) The dose and effect data were obtained from MTT assay (average value of triplicate) and were subjected to CompuSyn analysis. (II) Parameters were calculated from the median-effect equation and the median-effect plot. The " $\mathrm{m}$ " is the slope, which signifies shape; $\mathrm{Dm}$ is the $\mathrm{IC}_{50}$ value ( $\mathrm{AP}$ in $\mathrm{mM}, \mathrm{MP}$ in $\mathrm{mM}$ ) that signifies potency; and $\mathrm{r}$ is the linear correlation coefficient that signifies conformity. (III) Combination index (CI) was calculated from the CI equation algorithms using CompuSyn software. CI $=1,<1$ and $>1$ indicates additive effect, synergism and antagonism, respectively. (IV) The dose-reduction index (DRI) was calculated from the DRI equation and algorithm using CompuSyn software. 


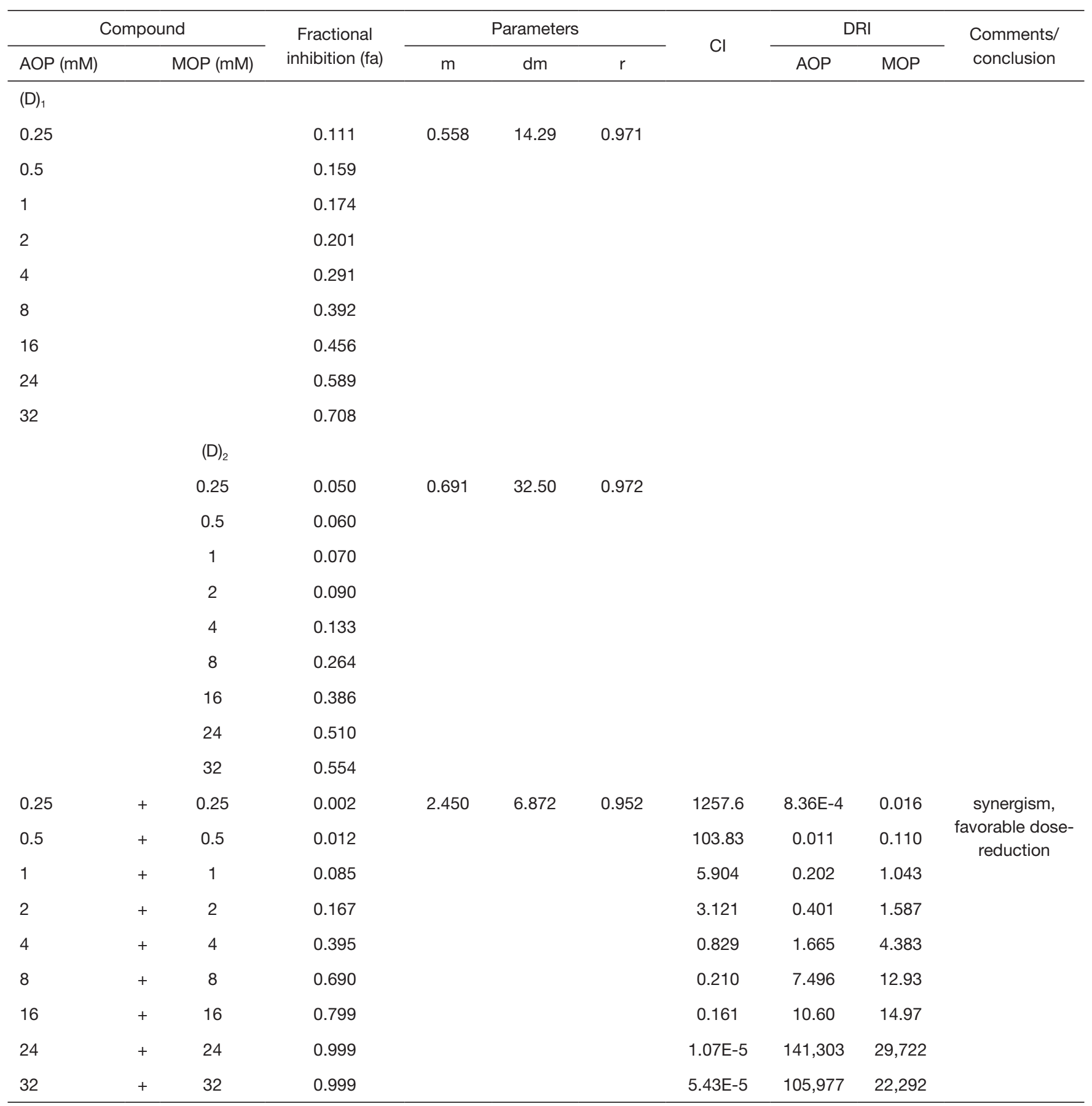

Figure S3 Combination of ALA-OPDT (AOP) and MET-OPDT (MOP) against MDA-MB-231 cell growth in MTT assay in vitro. The table is constructed by the contents generated from CompuSyn Report. (I) The dose and effect data were obtained from MTT assay (average value of triplicate) and were subjected to CompuSyn analysis. (II) Parameters were calculated from the median-effect equation and the median-effect plot. The " $\mathrm{m}$ " is the slope, which signifies shape; $\mathrm{Dm}$ is the $\mathrm{IC}_{50}$ value (AOP in $\mathrm{mM}, \mathrm{MOP}$ in $\mathrm{mM}$ ) that signifies potency; and $\mathrm{r}$ is the linear correlation coefficient that signifies conformity. (III) Combination index (CI) was calculated from the CI equation algorithms using CompuSyn software. CI $=1,<1$ and $>1$ indicates additive effect, synergism and antagonism, respectively. (IV) The dose-reduction index (DRI) was calculated from the DRI equation and algorithm using CompuSyn software. 